\title{
Model of Supplying Equipment System Based on the Assessment of the Residual Resource of Mining Drilling Rigs Components
}

\author{
Fares Abu-Abed ${ }^{1}{ }^{*}$ \\ ${ }^{1}$ Tver State Technical University, 17002622 A. Nikitin Street, Tver, Russia
}

\begin{abstract}
The structure of the rig includes many complex mechanisms their wear, and the value of the residual life depending on it, directly depend on the operating conditions of the rig. In compliance with all the norms and technical conditions of drilling and the absence of abnormal situations in its process, all components must develop their resource, defined by technical conditions, after which they need to be replaced. When various types of complications and abnormal situations occur in the drilling process, the load on the drilling machinery mechanisms can increase dramatically, which does not necessarily lead to the drill failure, but it affects the residual life of those mechanisms and components, the loads that exceed the standard values. With subsequent occurrences of similar situations in the drilling process, this may lead to failure of the drill. The article presents the rationale for choosing a simulation model for organizing a system for supplying drilling rigs with spare parts and accessories based on the theory of queuing systems. The work is an integral part of previously published developments presented in the materials of articles in 2-nd and 3-rd International innovative mining symposiums (2017-2018).
\end{abstract}

\section{Introduction}

Assessing the impact of an abnormal or pre-emergency situation on the magnitude of the residual resource is a difficult and poorly formalized task [1]. Therefore, to determine changes in the residual life of components of a drilling rig during its operation, the method of expert assessments can be used [2].

The set of such estimates can be represented as a matrix A (C, K) (Figure 1), where the element a $[\mathrm{i}, \mathrm{j}]$ represents the relative decrease in the residual life of the component $\mathrm{Kj}$ in the event of a pre-emergency situation $\mathrm{Ci}$. That is, when a situation occurs $\mathrm{Ci}$, the residual life of the drill components is changed as follows:

$$
\begin{aligned}
& \text { Residual resource }(K j) \text { Pre }- \text { crash situation }= \\
& \text { Residual resource }(K j) \times(1-a[i, j])
\end{aligned}
$$

\footnotetext{
*Corresponding author: aafares@mail.ru
} 


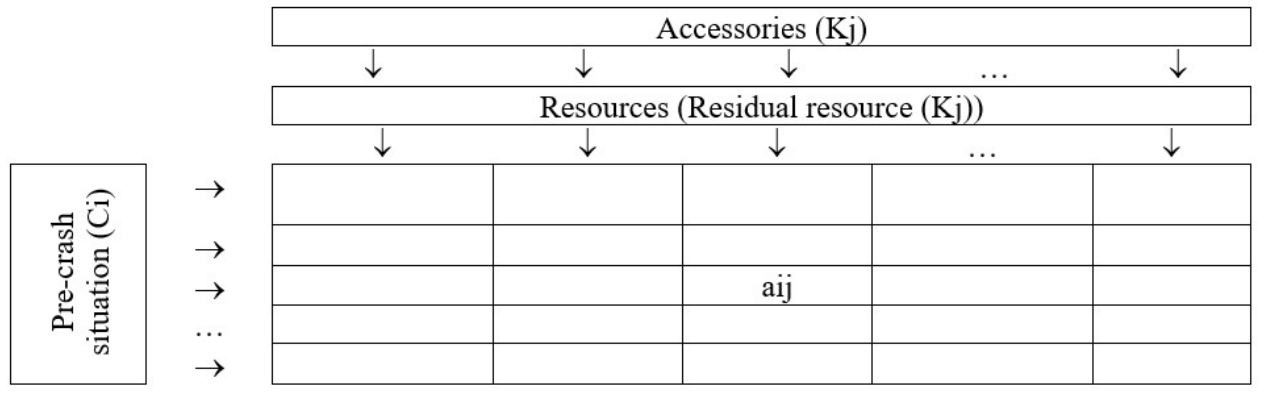

Fig. 1. The matrix of the coefficients of the component resource change in the event of a preemergency situation.

\section{Materials and Methods}

Rigs use different types of stock items. By appointment, there are three types of spare parts kit [2-6]. A single spare parts kit (KIT-S) is designed to restore the working capacity of a single drilling rig and is located near the place of its operation in order to ensure a short replacement time (several tens of minutes). The initial inventory level of the KIT-S set is replenished in accordance with accepted rules from the replenishment source (RS). When several drilling rigs are operated simultaneously (usually at least three), a group spare parts kit (KIT-G) can be created, which is equally accessible to any of the drilling rigs. The main difference between KIT-S and KIT-G consists in a significantly longer delivery time of the spare parts to the place of operation of the drilling rig in which the failure occurred. Therefore, the creation of a KIT-G is possible in two cases: the allowable break time is rather long, but significantly less than the time interval between adjacent failures; for all types of modules presented in the KIT-G, the system has internal structural redundancy. The KIT-G is also updated from the RS.

If several drilling rigs are in operation, but the necessary conditions for creating spare parts and equipment for one or several types of interchangeable modules are not fulfilled, then a two-level spare parts system (spare parts-2L) is created. At the first (lower) level, sets of KIT-S are placed, at the second (upper) level - a set of KIT-G. The KIT-G is replenished from the RS, and the KIT-S can be replenished either from the KIT-G, if it contains the same type of replacement part, or directly from the RS, if this type of replacement part is not in the KIT-G [7].

Various methods can be used to replenish spares kit. In case of periodic replenishment (PR), the replenishment period $\mathrm{Ti}$ of the $\mathrm{i}$-th type is assigned, after which the reserves are replenished to the initial level. If stocks are exhausted before $\mathrm{Ti}$ and another failure has occurred, the system goes into inoperable state until the next replenishment. When replenishing with emergency deliveries (ED), a period Ti is also assigned. However, in this case, in contrast to the PR, after the stocks are exhausted and the next failure, an emergency delivery of spare parts is organized to replace the failed module and replenish the stocks to the initial level. This reduces the average waiting time for recovery. When replenishing at a given level (GL), a request for replenishment of stocks is formed after the reserves are reduced to the established level, including zero, before the next failure occurs. The application is always formed when the system is operational. This way GL differs from ED. The GL method is characterized by a threshold stock level of components and distribution (or average value) of delivery time $[3,6]$. In the case of continuous replenishment (CR), a request for replenishment of stocks is formed and submitted for execution after each failure and use of each spare part. The CR method is characterized by the distribution or average value of the delivery time of the spare part. 


\section{Results and Discussion}

Supply systems typically operate in an environment of uncertainty. In the management of material flows factors must be taken into account, many of which are random. Under these conditions, the creation of an analytical model that establishes clear quantitative relationships between the various components of logistic processes may be either impossible or too expensive [4].

The system of supplying spare parts, tools and accessories (KIT) of drilling rigs (DR) means a multi-level system consisting of a set of single, group sets of operational spare parts and spare parts and stock of the source. The components of the system are interconnected and have certain characteristics. The spare parts system is designed to restore the performance of the DR after failures during operation. Consider a two-level system of stocks of elements with periodic replenishment, emergency deliveries and repair of failed elements in the repair agencies [8-10].

Simulation includes two main processes: the first is the construction of a model of a real system, the second is the formulation of experiments on this model.

The following objectives can be pursued:

a) understand the behavior of the logistics system;

b) choose a strategy that ensures the most efficient operation of the logistics system.

Systems of drilling rigs and their serving warehouses of spare parts and equipment are dynamic, that is, their parameters change over time. Therefore, the state of the system, the properties of the object and the number of active objects, parameters, actions and delays are all functions of time and are constantly changing in the process of modeling.

However, in the process of modeling, discrete points in time can be distinguished when events occur that reflect the sequence of changes in the system states over time. If the behavior of the system at other points in time is not fundamentally important, then we can use the technology of discrete-event modeling. Such modeling is called imitational.

Queue systems (QS) with a large number of application generators and service devices often have a structure that can be represented as a network. In the general case, a QS network is a graph whose vertices are single-channel and multichannel QS (arcs define demand transfer streams) [9].

When simulating the maintenance and repair work of drilling rigs, it is advisable to use the network QS apparatus in the event that the repair work is carried out by the crews located in the KIT storage points. In this case, we can distinguish two cases:

1. Each drill has its own fixed item of a group spares, which is responsible for its completion. At the same time, each item of the group spares has a fixed set of drilling rigs, which it supplies. In this case, the QS network degenerates into a set of closed subnets.

2. Each drilling site may apply to several items of spare parts (in the limit - to any such item) that can either accept or reject the application for a component.

The choice of one or another method of formalization of the existing drilling supply network depends on the actual topology of the service network. The second case is the most common.

Figure 2 shows a simulation model of a rig complex in the form of a queuing network, depending on the structure of which, and on the number and intensity of requests, the characteristics of applications of individual classes and for the combined flow are determined. 


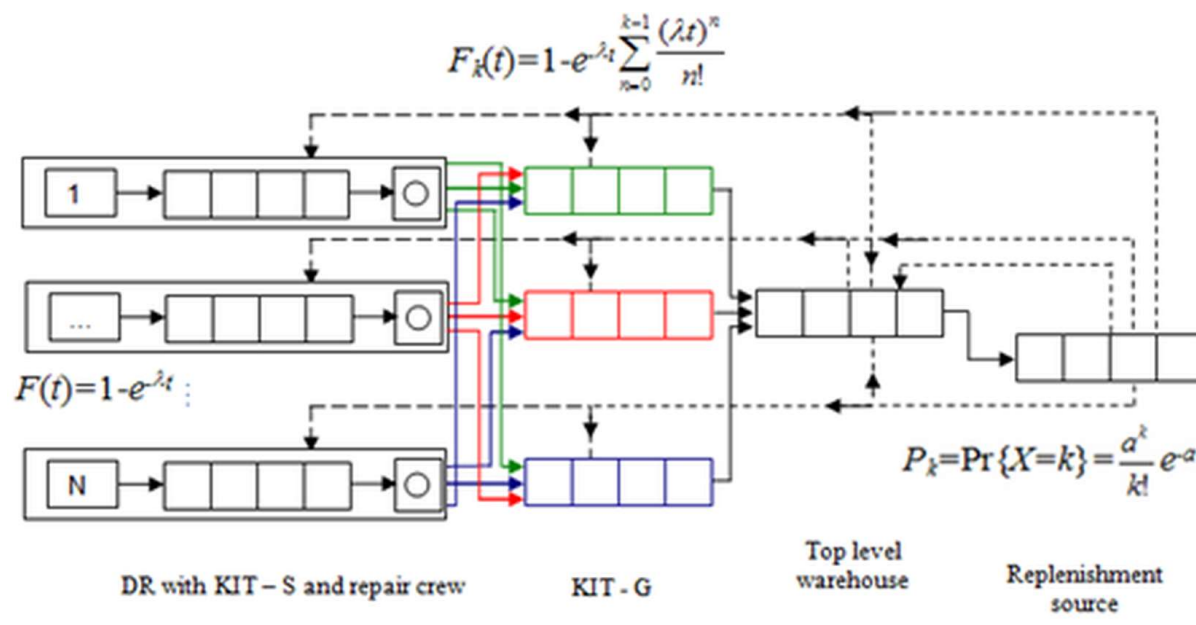

Fig. 2. Model of the supplying network of drilling parts.

Drilling rigs, KIT-S, KIT-G (each of which is associated with several sources of applications), act as nodes - sources of requests for parts, and repair crews - as service nodes.

Requirements (requests) from drilling rigs for components for which the value of the residual resource is reduced to a threshold value are received at intervals that are exponentially distributed $\left(F(t)=1-e^{-\lambda t}\right.$, гдe $\left.t \geq 0\right)$ with intensity (parameter of exponential value) $\lambda$ and coefficient of variation $v=1$ [5], forming the simplest flows on a single spare parts (KIT-S), which is located directly on the rig.

If several parts fail in the same rig, then the model parameterization includes the following transformations of the initial parameters (it is assumed that all input streams are the simplest):

1) $\Lambda=\sum_{k=1}^{H} \lambda_{k}$ - the intensity of the combined stream (the simplest);

2) $B=\sum_{k=1}^{H}\left(\frac{\lambda_{k}}{\Lambda}\right) b_{k}$ - averaged time to serve combined stream applications, where $\frac{\lambda_{k}}{\Lambda}$ is the share of class $\mathrm{k}$ applications in the total flow $(k=\overline{1, H})$;

3) $\Lambda B^{2}\left(1+v^{2}\right)=\sum_{k=1}^{H} \lambda_{k} b_{k}^{2}\left(1+v_{k}^{2}\right)$ - from this expression is determined by the coefficient of variation of the duration of service applications of the combined stream.

A graphic representation of the admission process may be as follows:

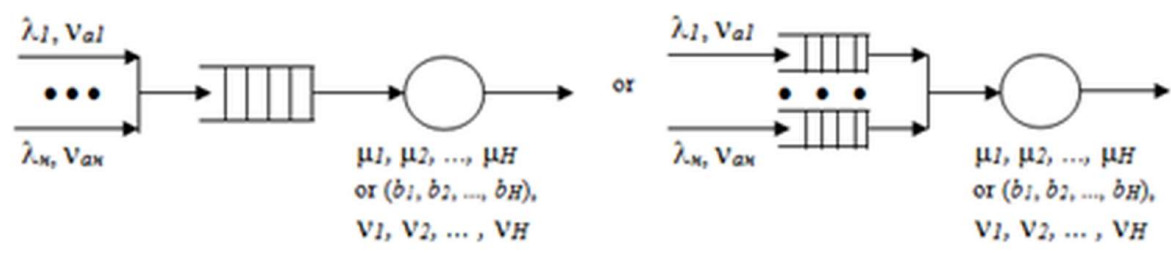

Fig. 3. The process of receipt of applications for components.

The outlined provisions are summarized in the flowchart of the simulation model algorithm (Figure 4). 


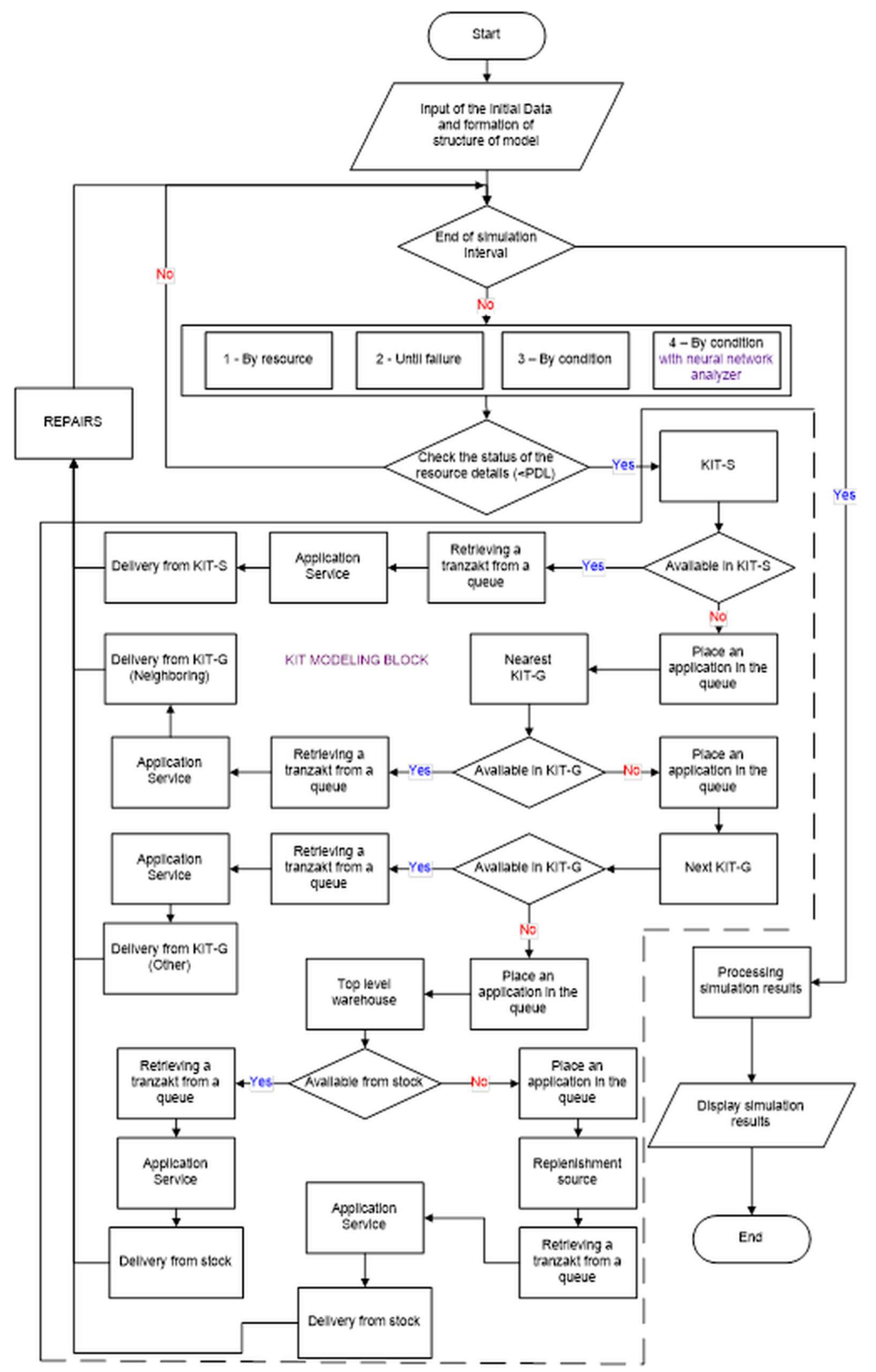

Fig. 4. Block diagram of the simulation model. 


\section{Conclusion}

As blocks of the simulation model for this subject area, you must implement:

- Block generating events associated with abnormal situations at the rig. This block creates a dynamic object - a repair requirement, in which the name of the failed component is recorded; - The assignment block to the object of the requirement of another dynamic object - a spare parts store, which must satisfy the request for the failed component;

- Requirements service unit, if necessary (in the case of a group spares) creates a requirement for the transportation of components from a group spares to the rig;

- The assignment block for the requirement to transport a dynamic transport facility that will deliver the component to the drilling rig;

- Repair operations block that implements the planned action - restoration of the drilling rig after delivery of the required component.

The effective analysis of the work of the model presented above makes it possible to consider it reasonable that it is possible to use the results of recognition of pre-emergency situations to improve the efficiency of the supply system with spare parts and accessories of drilling rigs by assessing the residual life of components in the event of pre-emergency situations.

This becomes possible given the previously conducted experimental studies:

1. Influence of neural network parameters on the effectiveness of learning to recognize pre-emergency situations.

The accuracy of recognition of pre-emergency situations and the number of iterations necessary for the convergence of the algorithm were used as estimates of the effectiveness of the modernized learning algorithm [8].

2. Influence of the parameters of the modified learning algorithm of the neural network to recognize pre-emergency situations on the efficiency of its work [11].

\section{References}

1. F. Abu-Abed., A. Khabarov, JFAS, 9:2S., 1171-1181 (2017)

2. Drilling Technologies (Universita Campus Bio-Medico, Roma, 2017)

3. M. Alrumah, T. Ertekin, J. Pet. Gas Eng., 10:2, 14-22 (2019)

4. European Standards References: EN 16228-1 Drilling and foundation equipment-Safety, Part 1-Part 7 (ESA, Brussels, 2016)

5. Rotary Foundation Drilling Rig Safety (OAFS, Paris, 2016)

6. Prudent development: Realizing the Potential of North America's Abundant Natural Gas and Oil Resources (OPC, Oslo, 2016)

7. F. Abu-Abed, A. Khabarov, E3S Web Conf., 41, 01028 (2018)

8. F. Abu-Abed, N. Borisov, E3S Web Conf., 21, 01019 (2017)

9. F.N. Abu-Abed, Oil Industry, 21, 100-102 (2014)

10. F.N. Abu-Abed, Oil Industry, 11, 107-111 (2015)

11. F. Abu-Abed, E3S Web Conf., 41, 01025 (2018) 